\title{
Plasmodium falciparum cGMP-Dependent Protein Kinase - A Novel Chemotherapeutic Target
}

\author{
David Rotella ${ }^{1}$, John Siekierka ${ }^{1}$ and Purnima Bhanot ${ }^{2 *}$ \\ ${ }^{1}$ Department of Chemistry and Biochemistry, Montclair State University, Montclair, NJ, United States, ${ }^{2}$ Department of \\ Microbiology, Biochemistry and Molecular Genetics, Rutgers New Jersey Medical School, Newark, NJ, United States
}

The primary effector of cGMP signaling in Plasmodium is the cGMP-dependent protein kinase (PKG). Work in human-infective Plasmodium falciparum and rodent-infective Plasmodium berghei has provided biological validation of $P$. falciparum PKG (PfPKG) as a drug target for treating and/or protecting against malaria. PfPKG is essential in the asexual erythrocytic and sexual cycles as well as the pre-erythrocytic cycle. Medicinal chemistry efforts, both target-based and phenotype-based, have targeted PfPKG in the past few years. This review provides a brief overview of their results and challenges.

OPEN ACCESS

Edited by:

Annette Elizabeth Kaiser, University of Duisburg-Essen,

Germany

Reviewed by:

Friedrich Wilhelm Herberg, University of Kassel, Germany

Satish Mishra,

Central Drug Research Institute

(CSIR), India

*Correspondence:

Purnima Bhanot

bhanotpu@njms.rutgers.edu

Specialty section:

This article was submitted to Antimicrobials, Resistance and Chemotherapy,

a section of the journal

Frontiers in Microbiology

Received: 25 September 2020 Accepted: 16 December 2020 Published: 03 February 2021

Citation:

Rotella D, Siekierka J and Bhanot P (2021) Plasmodium falciparum cGMP-Dependent Protein Kinase - A Novel Chemotherapeutic Target. Front. Microbiol. 11:610408. doi: 10.3389/fmicb.2020.610408
Keywords: Plasmodium, malaria, cGMP signaling, second messenger, kinase

\section{PKG IS THE KEY MEDIATOR OF cGMP SIGNALING IN Plasmodium}

cGMP is a second-messenger in eukaryotic cells. It is synthesized from GTP by guanylate cyclases and hydrolyzed by phosphodiesterases. Changes in intracellular levels of cGMP are converted to cellular responses through the action of effector proteins such as cGMP-dependent protein kinases [also known as protein kinase G (PKG)], cGMP-gated ion channels and cGMP binding proteins. Plasmodium genomes encode single copies of PKG and a putative cGMP-binding protein but the latter lacks key residues required for cyclic nucleotide binding (Moon et al., 2009). There are no homologs of cGMP-gated ion channels in the genome. PKG is likely to be the key mediator of cGMP signaling in the parasite.

cGMP-dependent protein kinase consists of a carboxy terminus regulatory domain fused to a kinase domain. The regulatory domain of Plasmodium (and other Apicomplexan) PKGs contain four cGMP binding sites, with one of them being degenerate and incapable of binding cGMP. In contrast mammalian PKGs have two cGMP binding sites. Another difference between mammalian and Plasmodium PKGs is while the former dimerizes, the latter is found as a monomer. Mammalian PKG is regulated through the combined action of an autoinhibitory segment present in the kinase's amino domain and by cGMP binding. In conditions of low cGMP, its substrate site is occupied by an autoinhibitory segment (Wall et al., 2003; Alverdi et al., 2008). Increasing cGMP levels lead to allosteric and cooperative occupation of the cGMP-binding sites in the regulatory domain, lifting the autoinhibition and activating the kinase domain. The kinase then phosphorylates substrate proteins on Ser or Thr residues. Regulation of Plasmodium PKG also requires cGMP binding (Kim et al., 2015; El Bakkouri et al., 2019; Byun et al., 2020) but, in a difference from mammalian PKG, not its putative autoinhibitory segment (Franz et al., 2018). PKG-dependent phosphorylation was detected in almost a 100 proteins in P. falciparum's erythrocytic stages (Alam et al., 2015; Vanaerschot et al., 2020) and in 193 proteins in P. berghei ookinetes (Brochet et al., 2014). 
The diversity of substrates indicates the variety of cellular pathways regulated by PKG. Interestingly, $P$. falciparum PKG (PfPKG) has a substrate-site preference that is substantially different from its mammalian homolog (Govindasamy et al., 2019).

\section{PKG FUNCTIONS IN EGRESS OF ERYTHROCYTIC MEROZOITES}

Conditional and chemical genetics have established the essential role of PKG in the asexual cycle (Taylor et al., 2010), specifically in the exit of merozoites from schizonts (Kim et al., 2015; Ganter et al., 2017). In P. falciparum schizonts PKG regulates the timely release of the protease SUB1 from exonemes into the parasitophorous vacuole and of AMA1 from micronemes to the merozoite surface (Collins et al., 2013). The net result of inhibiting PfPKG is a block in merozoite egress and interruption of the asexual cycle. The underlying mechanism of PfPKG's action is its regulation of phosphoinositide metabolism and consequently $\mathrm{Ca}^{2+}$ mobilization in the parasite (Brochet et al., 2014; recently reviewed in Brochet and Billker, 2016). In the related Apicomplexan, Toxoplasma gondii there is evidence that PKG-regulated egress of parasites is antagonized by cAMP signaling mediated by the parasite's cAMP dependent protein kinase pathways (Jia et al., 2017). Chemical inhibition of T. gondii PKG blocks parasite egress induced through genetic downregulation of $T$. gondii PKA signaling. Similar interplay between PKG and PKA pathways in Plasmodium has not yet been reported although $P$. falciparum $\mathrm{PKA}$ is essential for merozoite invasion (Wilde et al., 2019).

\section{PKG IS REQUIRED FOR GAMETOCYTE ACTIVATION AND OOKINETE MOTILITY}

In the mosquito midgut, activation of Plasmodium gametocytes to form gametes requires PKG. Its inhibition prevents the rounding up of gametocytes, an early step in gametocyte activation (McRobert et al., 2008). In mature ookinetes, PKG function is required for motility a prerequisite to ookinete invasion of the midgut (Brochet et al., 2014). As in asexual stages, in gametocytes and ookinetes PKG mobilizes intracellular $\mathrm{Ca}^{2+}$ and regulates vesicular traffic (Brochet et al., 2014). PKGdependent phosphorylation of proteins that are part of the actinomyosin motor also likely contributes to its regulation of parasite motility (Brochet et al., 2014; Govindasamy et al., 2019).

\section{PKG IS ESSENTIAL FOR PARASITE INVASION OF AND EXIT FROM HEPATOCYTES}

Conditional and chemical genetic approaches demonstrated that PKG plays a dual role in the pre-erythrocytic cycle. It is required for sporozoite motility and hence their invasion of hepatocytes for as well as for the formation and/or release of merosomes from infected hepatocytes. PKG's effect on sporozoite motility is mediated through the release onto the sporozoite surface of micronemal adhesins, such as TRAP (Govindasamy et al., 2016), which enable parasite attachment to the extracellular surface. Later during liver stage development, chemical inhibition of PKG or depletion of the protein prevents the formation and/or release of merosomes, membrane-bound packets of hepatic merozoites that are extruded from the infected hepatocyte to initiate the transition from the liver cycle to the erythrocytic cycle (Falae et al., 2010; Govindasamy et al., 2016; Govindasamy and Bhanot, 2020).

\section{TARGETING $P$. falciparum PKG IN THE ASEXUAL CYCLE}

The essential function of PfPKG in schizogony provided biological validation for its development as a drug target for treating the pathology-causing erythrocytic stages of $P$. falciparum. It is a new target in the global malaria drug discovery landscape. Inhibitors of PfPKG represent a novel mechanism of action since, based on literature precedent, none of the current compounds under development target PfPKG (Wells et al., 2015). Therefore, cross-resistance with existing agents is less likely. Medicinal chemistry programs directed against PfPKG have focused on activity against asexual stages in the blood and gametocytes in mosquitoes (Matralis et al., 2019; Penzo et al., 2019).

Plasmodium PKG is significantly different from mammalian PKG to allow specific targeting. A trisubstituted pyrrole, (1) (also called TSP) is a potent and selective inhibitor of PKGs of several Apicomplexan parasites (Diaz et al., 2006). It has an $\mathrm{IC}_{50}$ of 3.5 nM against PfPKG (Diaz et al., 2006; McRobert et al., 2008) and $2 \mu \mathrm{M}$ against human PKG (our unpublished data). This significant selectivity is associated with a larger "gatekeeper" residue, Gln in human PKG (Gurnett et al., 2002). PfPKG contains Thr at the "gatekeeper" position (amino acid 618) and its shorter side-chain allows access to compounds excluded from the smaller cavity in the human enzyme. Replacement of Thr618 in PfPKG with Gln (T618Q) leads to a 3000-fold increase in $\mathrm{IC}_{50}$ of the mutant enzyme against $\mathbf{1}$ (McRobert et al., 2008). Merck researchers pursued $\mathbf{1}$ and the imidazopyridine $\mathbf{2}$ as lead compounds for a veterinary medicine campaign directed against PKG of a related parasite, Eimeria tenella (Biftu et al., 2005, 2006). They succeeded in identifying lead subnanomolar compounds in both the TSP series, $\mathbf{1}$ and the imidazopyridine series, $\mathbf{2}$ but the program was ultimately discontinued due to Ames positive data and genotoxicity of the two top imidazopyridine compounds (Biftu et al., 2006). An imidazo (Wall et al., 2003; Moon et al., 2009) thiazole series was subsequently investigated as an alternative. This research took advantage of established structure activity information from previous work by this group that identified optimal 4-fluorophenyl and aminopyrimidine substituents on the heterocycle. A narrow range of amideand amino-derived possibilities was examined at the remaining thiazole position. In this group, amines were substantially more potent compared to the corresponding amide analog. This identified a derivative (3) with potent in vitro $\mathrm{PKG}$ activity $\left(\mathrm{IC}_{50}\right.$ 
$80 \mathrm{pM}$ ) and broad-spectrum in vivo potency in anticoccidial assays (Scribner et al., 2008).
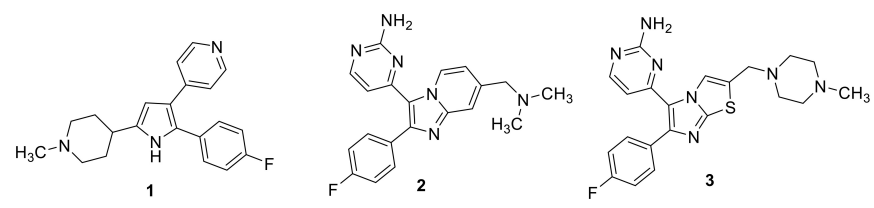

Drug discovery and PfPKG inhibitor optimization will be assisted by the availability of crystal structures of both P. falciparum and Plasmodium vivax PKG (El Bakkouri et al., 2019). This structure of the apoprotein showed how regions of the protein are arranged in the inactive structure. Based on this information, the team proposed a mechanism for activation by cGMP binding that may prove useful in further biochemical studies.

In an important paper that provided direct preclinical validation of PfPKG as a target for antimalarial therapy, Baker et al. (2017) reported that an imidazopyridine based PfPKG inhibitor could clear P. falciparum infection in mice engrafted with human erythrocytes. This molecule, 4 (ML10), resulted from a structure-activity study that was aided by structurebased design. Specific hydrogen bonding interactions between the sulfonamide substituent in the pendant aryl ring with Asp675 and Phe670 in the enzyme, along with key hinge region interactions between Val614 and the pyrimidine moiety contributed to excellent in vitro potency ( $\mathrm{IC}_{50} 160 \mathrm{pM}$ ) that translated very well to cell based studies $\left(\mathrm{EC}_{50} 2 \mathrm{nM}\right)$ and high selectivity in a panel of human kinases. As expected, ML10 was several orders of magnitude less potent in vitro against the "gatekeeper" mutant PfPKG (T618Q) and similarly less active in culture of asexual stage parasites expressing the mutant enzyme. Oral administration of 4 to mice at a dose of $25 \mathrm{mg} / \mathrm{kg}$ twice daily reduced $P$. berghei parasitemia by $50-60 \%$ over 4 days. A single $50 \mathrm{mg} / \mathrm{kg}$ dose of 4 reduced Plasmodium chabaudi infection by approximately $50 \%$ in mice and two $50 \mathrm{mg} / \mathrm{kg}$ doses in one day was as effective as a single $10 \mathrm{mg} / \mathrm{kg}$ dose of chloroquine in this model. Greater efficacy against $P$. chabaudi was attributed to its synchronized asexual cycle, because of which the twice-daily dosing was able to span the entire merozoite egress-reinvasion window. Using blood stage $P$. falciparum in mice, $\mathbf{4}$ was dosed at 50 or $100 \mathrm{mg} / \mathrm{kg}$ for 4 days, reduced parasitemia substantially in a dose response manner. Interestingly, attempts to generate $P$. falciparum strains resistant to $\mathbf{4}$ in culture were unsuccessful. Other cell culture experiments showed that $\mathbf{4}$ had no cytotoxic effect on three different human cell lines at concentrations as high as $10 \mu \mathrm{M}$.
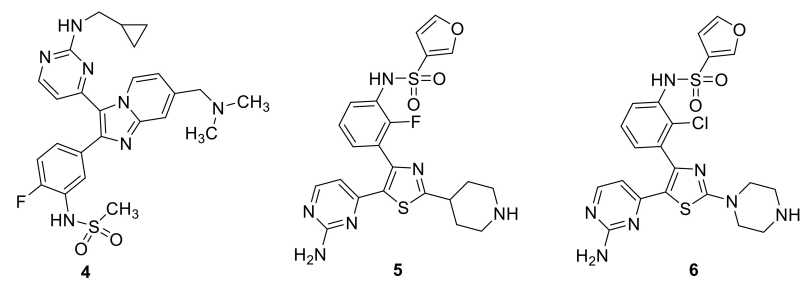

Penzo et al. (2019) carried out a high throughput screen of a 1.7 million compound library provided by GlaxoSmithKline. This screen identified a number of hit scaffolds that included imidazoles, pyrazoles, and thiazoles. The group chose to focus on thiazoles because previous experience suggested this template offered the greatest potential for improved selectivity among human kinases. Chemoproteomic experiments with thiazole examples identified in the screen revealed that in addition to potent PfPKG inhibition ( $\mathrm{IC}_{50} \sim 1 \mathrm{nM}$ ), some of these compounds also inhibited CDPK1, NEK1, CDPK4, CK1, and CRK5. In a 48-h P. falciparum growth inhibition assay, two of these compounds, 5 and $\mathbf{6}$, gave good activity, indicative of a desired fast killing profile. It was equally interesting to observe that these compounds were equally active against $P$. falciparum parasites that expressed a T618Q mutant PfPKG. The hits were substantially less potent in vitro against the mutant enzyme, suggesting that these compounds are acting at one or more other targets in the parasite.

The Baker group reported progress in the optimization of thiazoles in 2018 and interest in them may have been stimulated by a combination of the Ames results and potential for good kinase selectivity noted above (Tsagris et al., 2018). The team applied established structure-activity information associated with $\mathbf{1}$ as a starting point for their work. The monosubstituted pyridine in $\mathbf{1}$ is a likely site for metabolism limiting oral bioavailability and as a result, alternatives were sought. An aminopyrimidine replacement 7 was identified with good in vitro PfPKG potency (IC $5017 \mathrm{nM}$ ), but measurably weaker cellular activity $\left(\mathrm{EC}_{50}\right.$ $\sim 1800 \mathrm{nM}$ ), compared to 1 . Substituents were added to the primary amino group and this resulted in phenyl piperazine derivative 8 with improved PfPKG activity (IC 50300 pM) and excellent cellular activity $\left(\mathrm{EC}_{50} 25 \mathrm{nM}\right)$. Using the binding model developed earlier, it was hypothesized the phenyl piperazine filled a hydrophobic pocket more completely. However, this compound was not as selective as $\mathbf{6}$ and modifications to the 4-fluorophenyl moiety were evaluated. This resulted in identification of 9, a highly selective PfPKG inhibitor with slightly lower cellular efficacy compared to 8 (EC $50115 \mathrm{nM})$.
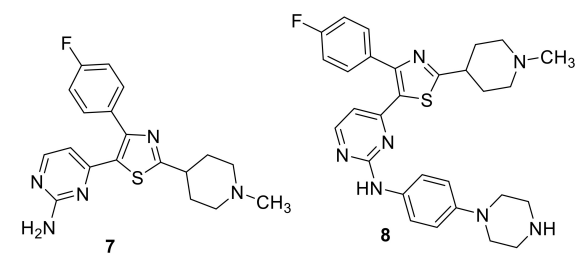

More recently, the Baker group disclosed the identification of novel trisubstituted thiazoles that exhibited rapid parasite killing properties (Matralis et al., 2019). There is a need in the field to develop such rapid killing compounds because of increasing resistance to artemisin-class antimalarials. This article revealed that a highly selective PfPKG inhibitor such as 9 exhibited slow parasite killing kinetics. The team focused on pyrimidinyl nitrogen substituents because previous experience showed that this position played a key role in PfPKG potency. This therapeutic 
hypothesis stimulated the team to focus primarily on wholecell activity rather than potency against PfPKG and, following development principles, kept other important pharmaceutical properties in mind such as hERG, cytotoxicity, metabolic clearance rate and water solubility. Cell culture experiments measured kinetic effects on $P$. falciparum killing and new compounds were compared using artesunate (an artemisinin analog), pyrimethamine and atovaquone as positive controls to provide kinetic range in the assay. Transmission blocking activity was measured by assessment of exflagellation of male gametocytes and by expression of Pfs 25 protein expression in female gametocytes. There is substantial evidence that PfPKG plays an essential role in gametogenesis. Two compounds were highlighted in this paper, 10 and 11. While $\mathbf{1 0}$ had unfavorable hERG activity ( $\left.\mathrm{IC}_{50} 1 \mu \mathrm{M}\right)$, it rapidly killed $P$. falciparum in culture, exhibiting a rate and extent $(>90 \%$ at $24 \mathrm{~h}$ ) comparable to artesunate. In addition, $\mathbf{1 0}$ blocked transmission with $\mathrm{EC}_{50}$ values of 300 and $400 \mathrm{nM}$, respectively in male and female gametocyte assays. Furthermore, 10 was equally efficacious when tested in culture using wild-type and T618Q mutant $P$. falciparum with $\mathrm{EC}_{50}$ values approximately 150 nM. Further SAR led to the identification of 11 with comparable fast killing properties and lower hERG activity ( $\mathrm{IC}_{50} 4 \mu \mathrm{M}$ ). Compound $\mathbf{1 0}$ was employed in chemoproteomic experiments to identify other proteins that might either contribute to its rapid killing activity and/or provide new targets for future investigation. These experiments highlighted a serine/arginine protein kinase, SRPK2, as a candidate for further investigation.
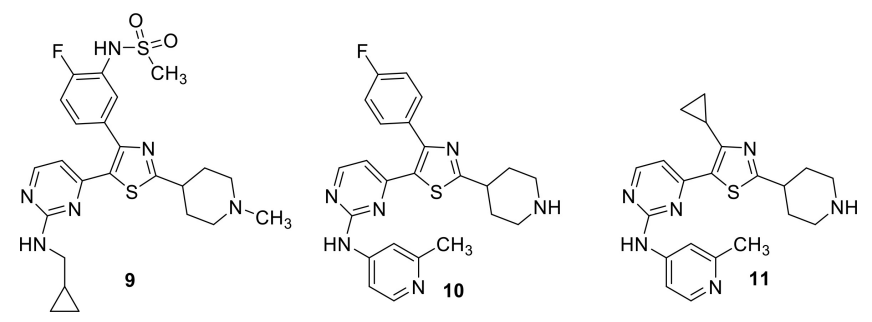

Another compound, MMV030084 (12) targeting PfPKG was found through phenotypic screening using sporozoite invasion and asexual stage growth assays (Vanaerschot et al., 2020). It also blocks male gametogenesis. Chemoproteomics was then used to determine that 12 interacts with PfPKG. Conditional depletion of PfPKG protein in $P$. falciparum asexual parasites decreased the $\mathrm{IC}_{50}$ of the compound by fourfold, consistent with heightened sensitivity of the parasite to a compound when the compound's in vivo target is less abundant. Compound $\mathbf{1 2}$ is structurally similar to $\mathbf{1}$ and molecular docking studies suggest that it exploits the same binding pocket.

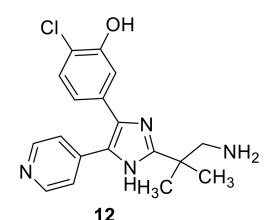

\section{TARGETING PfPKG FOR CHEMOPROTECTION}

The "Chemoprotection" against malaria target product profile is described as a combination of two or more drugs that are active against Plasmodium's hepatic schizonts and may also have activity against asexual blood stages (Burrows et al., 2017). Our data provide proof-of-principle that inhibition of the pre-erythrocytic cycle through specific and selective chemical targeting of Plasmodium PKG is possible, making it a target for development of chemoprotective drugs.

We have demonstrated that $\mathbf{1}$ targets $P$. berghei PKG (PbPKG) and blocks protein secretion and therefore parasite motility, invasion of hepatocytes and formation of liver stages by P. berghei sporozoites (Govindasamy et al., 2016). Treatment with 1 postinvasion leads to liver stages that are significantly smaller in size (our unpublished data). It also blocks maturation of liver schizonts into merosomes, membrane-bound packets of hepatic merozoites that are extruded from an infected hepatocyte into the bloodstream (Govindasamy et al., 2016). Liver stages that are developmentally arrested due to loss of PbPKG elicit a protective host immune response that protects from future infection by wild-type sporozoites (Falae et al., 2010). The block in merosome formation and/or release resulting from 1's inhibition of PbPKG (Govindasamy et al., 2016) is similar to the phenotype of P. berghei liver stages mutants missing PKG (Falae et al., 2010).

The $\mathrm{EC}_{50}$ of $\mathbf{1}$ against transgenic $P$. berghei sporozoites and liver stages expressing the "gatekeeper" PbPKG $\left(\mathrm{T}_{619} \mathrm{Q}\right)$ is significantly higher than against wild-type sporozoites $\left(\mathrm{EC}_{50}=2.12\right.$ vs. $\left.0.11 \mu \mathrm{M}\right)$ (Govindasamy et al., 2016). The decreased sensitivity of $\mathrm{T}_{619} \mathrm{Q}-\mathrm{PKG}$ sporozoites and liver stages to $\mathbf{1}$ demonstrates that its target in pre-erythrocytic stages is $\mathrm{PKG}$, and that $\mathrm{PKG}$ is essential for sporozoite infection and liver stage maturation. Importantly, mice treated with 1, before and shortly after sporozoite infection, experience a significant delay or abolishment in blood stage parasitemia (Panchal and Bhanot, 2010).

This work also clarified the seeming contradiction between PbPKG being l's primary target in sporozoites and our observations that $P$. berghei mutant sporozoites in which the PbPKG gene has been conditionally deleted infect HepG2 cells normally and remain sensitive to $\mathbf{1}$ (Falae et al., 2010). We demonstrated that conditional deletion of PbPKG in sporozoites does not significantly decrease the long-lived PbPKG protein (Govindasamy et al., 2016). The near-normal levels of PbPKG protein in mutant sporozoites enable normal infectivity and sensitivity to 1 .

The pre-erythrocytic cycle of Plasmodium could be particularly vulnerable to Plasmodium PKG inhibitors because the compounds would have two opportunities to block the cycle. The first block will prevent sporozoite infection of the liver. Parasites that escape the first block will be targeted during their 1-2 week long intrahepatic period by a second block inhibition of the development/exit of mature liver forms from the hepatocyte. Developmentally arrested liver stages will be targeted by the host immune response that can clear them, and potentially provide protection against subsequent infections. We 
believe that these aspects of PKG function, the significantly lower parasite load in the liver, and the single infection cycle in the liver make pre-erythrocytic stages vulnerable to Plasmodium PKG inhibitors. In addition, Plasmodium PKG inhibitors will target the erythrocytic cycle and transmission to mosquitoes since the kinase is essential for merozoite egress and invasion, and for gametogenesis (Baker et al., 2017).

\section{ASSAYS FOR PfPKG INHIBITION}

In vitro activity of PfPKG has been assayed using a variety of formats including microfluidic mobility shift assays (Baker et al., 2017), immobilized metal ion affinity-based fluorescence polarization (IMAP) assays (Mahmood et al., 2020) and an NADH/ATPase coupled assay (El Bakkouri et al., 2019). In the microfluidic mobility shift assay, kinase reactions are performed using a fluorescently labeled peptide substrate. Phosphorylated products are separated, based on charge differences, by capillary electrophoresis and detected by laser-induced fluorescence. This technology allows acquisition of enzymatic data in a kinetic fashion which enables simultaneous measurement of both product and substrate concentrations for each reaction. The IMAP technology is based on the specific, high-affinity interaction of trivalent metal-containing nanoparticles with phosphorylated substrates. During the course of a kinase reaction fluorescently labeled substrate is phosphorylated and binds to metal nanoparticles, resulting in a change in fluorescence polarization between free peptide and nanoparticle-associated phosphorylated peptide, a direct measure of kinase activity. The NADH/ATPase coupled assay is a photometric microtiter plate assay which measures the accumulation of ADP during a kinase reaction and is based on ADP recycling coupled to the oxidation of NADH. One advantage of this assay format is that it does not require fluorophore labeled substrates. All three assays lend themselves to high throughput screening and can be used for both kinetic analysis (for example to determine a compound's mode of inhibition) and endpoint analysis (single determination at a defined time point). The IMAP and NADH/ATPase coupled assays are single step or "homogeneous" assays with no requirement for separation of reaction products. All three assays were so far deployed at the $\mathrm{K}_{\mathrm{m}}$ of ATP and hence selected for ATP-competitive inhibitors of PfPKG. It is interesting to speculate that compound screens executed at saturating concentrations of ATP might identify compounds with novel modes of inhibition such as allosteric inhibitors of PfPKG.

Exploiting screening strategies to identify non-competitive or allosteric inhibitors could lead to the identification of novel inhibitors with enhanced selectivity. Screening compound libraries at high substrate concentrations for example, could bias a screen toward identifying non-competitive inhibitors. Other screening technologies such as ThermoFluor (Zhang and Monsma, 2010) could be utilized to identify noncompetitive inhibitors. ThermoFluor or differential scanning fluorimetry (DSF) assesses the direct binding of a ligand to its target protein by measuring the enhancement of the thermal stability of the target protein. The technique provides realtime analysis of ligand (compound) binding to a target protein. The structural differences between PfPKG and the human ortholog (number of cGMP binding domains and the role of the pseudosubstrate autoinhibitory sequence) may provide a conformational arrangement favorable to identifying unique allosteric inhibitory sites. ATP non-competitive inhibitors have been successfully identified for a variety of protein kinases such as the Bcr Abl kinase inhibitor, Gleevec which was the first successful therapy for chronic myelogenous leukemia (Druker et al., 1996). Recent work has demonstrated the potential for allosteric inhibition of PfPKG by targeting its key cGMP binding site (Byun et al., 2020).

The activity of PfPKG inhibitors in whole cells has been assayed in asexual stages, gametocytes and liver stages. For determining the effect on P. falciparum asexual stage growth, inhibitors are added to cultures composed primarily of ringstages (drug-sensitive 3D7 strain) at $t=0 \mathrm{~h}$ (Desjardins et al., 1979; Baker et al., 2017). Radioactive hypoxanthine is added to cultures at $48 \mathrm{~h}$, which corresponds to the time of parasite release from schizonts, and its incorporation into parasites is measured at $72 \mathrm{~h}$. This assay captures the effect of compounds on parasite growth in a $24 \mathrm{~h}$ cycle.

The in vivo efficacy of PfPKG inhibitors against asexual stages has been tested using $P$. berghei ANKA, $P$. chabaudi and a P. falciparum-humanized mouse model (Baker et al., 2017). Mice infected with $P$. berghei ANKA or P. chabaudi were treated with compounds for 4 consecutive days. Since $P$. chabaudi undergoes a synchronized intra-erythrocytic cycle, compound administration was timed to coincide with schizont rupture and merozoite reinvasion of RBCs. Parasitemias of treated and control mice were determined after 4 days of treatment. In vivo efficacy against $P$. falciparum asexual stages was determined in the GSKPfalcHuMouse (Jimenez-Diaz et al., 2009). These immunodeficient mice are engrafted with human erythrocytes to achieve $40 \%$ chimerism and then infected with P. falciparum 3D7 asexual stage parasites. Compound administration commences 3 days post-infection and parasitemias are determined after 4 days of consecutive treatment.

Effect of PfPKG inhibitors on male gametocytes has been determined by measuring exflagellation centers observed upon addition of xanthurenic acid to a NF54 gametocyte-containing culture incubated at about $27^{\circ} \mathrm{C}$ (Delves et al., 2013; Matralis et al., 2019). Activation of female gametocytes was assayed in the same culture using an antibody against Pfs 25 , a protein expressed on the surface of female gametes (Delves et al., 2013; Matralis et al., 2019). The effect of these inhibitors on parasite development in mosquitoes is assayed by microscopic counting of oocysts present in midguts of mosquitoes at day 7 post-feeding of a gametocyte bloodmeal (Baker et al., 2017).

Determining the efficacy of PfPKG inhibitors against preerythrocytic stages has utilized rodent-infective $P$. berghei ANKA sporozoites. Sporozoites obtained from salivary glands of infected mosquitoes are added to HepG2 cells, a cultured human hepatoma cell line, together with inhibitors. Inhibitorcontaining medium is replaced with inhibitor-free medium at 14-18 h post-infection. The number of infected HepG2 cells 
is determined at $40-48 \mathrm{~h}$ post-infection using immunostaining with a parasite-specific antibody or measurement of luciferase activity if luciferase-expressing sporozoites were used. Therefore this assay measures the effect of PfPKG inhibition on sporozoite entry into hepatocytes (Govindasamy et al., 2016). The in vivo efficacy of this inhibition was examined using $P$. berghei and Plasmodium yoelii-infected mice (Panchal and Bhanot, 2010). Sporozoites were delivered through either mosquito bite or intravenous injection. Compounds were administered prior to sporozoites and $2 \mathrm{~h}$ post-infection to cover a time-window spanning sporozoite exit from the site of bite and their invasion of the liver. Parasite burden in the liver was determined, at $40 \mathrm{~h}$ postinfection, by quantifying either parasite $18 \mathrm{~S}$ rRNA expression via real-time PCR or bioluminescence in the liver, if using luciferaseexpressing sporozoites, via in vivo intravital imaging (IVIS). The length of time between sporozoite inoculation and appearance of parasites in the blood (pre-patent period) can also used as an indirect measure of liver-cycle inhibition.

\section{SAFETY CONSIDERATIONS FOR TARGETING Plasmodium PKG}

Targeting kinases requires an extra consideration for toxicity. This risk can be minimized through rigorous incorporation of structural features that optimize affinity for the $P$. falciparum kinase and reduce affinity for human kinases. Development of top compounds requires human kinome-wide selectivity analysis to ensure selectivity and enhance safety. In a chemoprotective role, PfPKG inhibitors will be administered for weeks to a few months, rather than chronically as in some other disease indications that use kinase inhibitors, further minimizing the risk from potential off-target activity of a parasite kinase-targeted molecule.

\section{PARASITE RESISTANCE TO PfPKG INHIBITORS}

Two studies have investigated the potential for parasites to evolve resistance to PfPKG inhibitors. In (Baker et al., 2017), exposure of parasites cultures to sub-lethal doses of $2\left(3 \times \mathrm{IC}_{50}\right)$ for 60 days did not lead to selection of resistance. In (Vanaerschot et al., 2020), parasites were similarly subjected to sub-lethal doses of

\section{REFERENCES}

Alam, M. M., Solyakov, L., Bottrill, A. R., Flueck, C., Siddiqui, F. A., Singh, S., et al. (2015). Phosphoproteomics reveals malaria parasite Protein Kinase G as a signalling hub regulating egress and invasion. Nat. Commun. 6:7285.

Alverdi, V., Mazon, H., Versluis, C., Hemrika, W., Esposito, G., van den Heuvel, R., et al. (2008). cGMP-binding prepares PKG for substrate binding by disclosing the C-terminal domain. J. Mol. Biol. 375, 1380-1393. doi: 10.1016/j.jmb.2007. 11.053

Baker, D. A., Stewart, L. B., Large, J. M., Bowyer, P. W., Ansell, K. H., Jimenez-Diaz, M. B., et al. (2017). A potent series targeting the malarial cGMP-dependent protein kinase clears infection and blocks transmission. Nat. Commun. $8: 430$.
12 and parasites with low-level resistance were selected. Wholegenome sequencing of resistant parasites identified a mutation in the Tyrosine-kinase like protein 3 (TKL3) but none in PfPKG. Introduction of this mutation into WT parasites or knocking-out TKL3 caused a threefold shift in the $\mathrm{IC}_{50}$ for $\mathbf{1 2}$. However, 12 did not inhibit the activity of recombinant TLK3 in vitro, even at $500 \times \mathrm{IC}_{50}$. These data strongly suggest that TKL3 mediates resistance against $\mathbf{1 2}$ and is not its target in vivo. It is proposed that phosphorylation of an essential PfPKG substrate by TKL3 permits the parasite to escape the egress defect imposed by inhibition of PfPKG by 12.

\section{OTHER P. falciparum CYCLIC NUCLEOTIDE-DEPENDENT PROTEIN KINASES AS POTENTIAL DRUG TARGETS}

Plasmodium falciparum contains a single cAMP-dependent protein kinase (PfPKA), composed of catalytic and regulatory subunits. PfPKA has been shown to phosphorylate P. falciparum apical membrane antigen 1 (PfAMA1) located in the micronemes of P. falciparum merozoites and is critical for parasite invasion (Patel et al., 2019; Wilde et al., 2019). Asexual stages of parasites lacking PfPKA exhibit a profound reduction in growth along with a reduction in erythrocyte invasion. The interplay between PfPKG and PfPKA in liver invasion by sporozoites remains to be established and may have important therapeutic implications.

\section{AUTHOR CONTRIBUTIONS}

$\mathrm{PB}, \mathrm{JS}$, and DR wrote and edited the manuscript. DR provided the figures. All authors contributed to the article and approved the submitted version.

\section{FUNDING}

PB, JS, and DR acknowledged support from the National Institutes of Health award R01AI133633. PB acknowledged support from the United States Department of Defense award W81XWH2010386.

Biftu, T., Feng, D., Fisher, M., Liang, G. B., Qian, X., Scribner, A., et al. (2006) Synthesis and SAR studies of very potent imidazopyridine antiprotozoal agents. Bioorganic Med. Chem. Lett. 16, 2479-2483. doi: 10.1016/j.bmcl.2006.01.092

Biftu, T., Feng, D., Ponpipom, M., Girotra, N., Liang, G. B., Qian, X., et al. (2005). Synthesis and SAR of 2,3-diarylpyrrole inhibitors of parasite cGMP-dependent protein kinase as novel anticoccidial agents. Bioorganic Med. Chem. Lett. 15, 3296-3301. doi: 10.1016/j.bmcl.2005.04.060

Brochet, M., and Billker, O. (2016). Calcium signalling in malaria parasites. Mol. Microbiol. 100, 397-408. doi: 10.1111/mmi.13324

Brochet, M., Collins, M. O., Smith, T. K., Thompson, E., Sebastian, S., Volkmann, K., et al. (2014). Phosphoinositide metabolism links cGMP-dependent protein kinase $\mathrm{G}$ to essential $\mathrm{Ca}(2)(+)$ signals at key decision points in the life cycle of malaria parasites. PLoS Biol. 12:e1001806. doi: 10.1371/journal.pbio.1001806 
Burrows, J. N., Duparc, S., Gutteridge, W. E., Hooft, van Huijsduijnen, R., Kaszubska, W., et al. (2017). New developments in anti-malarial target candidate and product profiles. Malar. J. 16:26.

Byun, J. A., Van, K., Huang, J., Henning, P., Franz, E., Akimoto, M., et al. (2020). Mechanism of allosteric inhibition in the Plasmodium falciparum cGMPdependent protein kinase. J. Biol. Chem. 295, 8480-8491. doi: 10.1074/jbc. ra120.013070

Collins, C. R., Hackett, F., Strath, M., Penzo, M., Withers-Martinez, C., Baker, D. A., et al. (2013). Malaria parasite cGMP-dependent protein kinase regulates blood stage merozoite secretory organelle discharge and egress. PLoS Pathog 9:e1003344. doi: 10.1371/journal.ppat.1003344

Delves, M. J., Ruecker, A., Straschil, U., Lelievre, J., Marques, S., Lopez-Barragan, M. J., et al. (2013). Male and female Plasmodium falciparum mature gametocytes show different responses to antimalarial drugs. Antimicrob. Agents Chemother. 57, 3268-3274. doi: 10.1128/aac.00325-13

Desjardins, R. E., Canfield, C. J., Haynes, J. D., and Chulay, J. D. (1979). Quantitative assessment of antimalarial activity in vitro by a semiautomated microdilution technique. Antimicrob. Agents Chemother. 16, 710-718. doi: 10.1128/aac.16.6.710

Diaz, C. A., Allocco, J., Powles, M. A., Yeung, L., Donald, R. G., Anderson, J. W., et al. (2006). Characterization of Plasmodium falciparum cGMP-dependent protein kinase (PfPKG): antiparasitic activity of a PKG inhibitor. Mol. Biochem. Parasitol. 146, 78-88. doi: 10.1016/j.molbiopara.2005.10.020

Druker, B., Tamura, S., Buchdunger, E., Ohno, G., Segal, M., Fanning, S., et al. (1996). Effects of a selective inhibitor of the Abl tyrosine kinase on the growth of Bcr-Abl positive cells. Nat. Med. 2, 561-566. doi: 10.1038/nm0596-561

El Bakkouri, M., Kouidmi, I., Wernimont, A. K., Amani, M., Hutchinson, A., Loppnau, P., et al. (2019). Structures of the cGMP-dependent protein kinase in malaria parasites reveal a unique structural relay mechanism for activation. Proc. Natl. Acad. Sci. U S A. 116, 14164-14173. doi: 10.1073/pnas.1905558116

Falae, A., Combe, A., Amaladoss, A., Carvalho, T., Menard, R., and Bhanot, P. (2010). Role of Plasmodium berghei cGMP-dependent protein kinase in late liver stage development. J. Biol. Chem. 285, 3282-3288. doi: 10.1074/jbc.m109. 070367

Franz, E., Knape, M. J., and Herberg, F. W. (2018). cGMP binding domain d mediates a unique activation mechanism in Plasmodium falciparum PKG. ACS Infect. Dis. 4, 415-423. doi: 10.1021/acsinfecdis.7b00222

Ganter, M., Goldberg, J. M., Dvorin, J. D., Paulo, J. A., King, J. G., Tripathi, A. K., et al. (2017). Plasmodium falciparum CRK4 directs continuous rounds of DNA replication during schizogony. Nat. Microbiol. 2:17017.

Govindasamy, K., and Bhanot, P. (2020). Overlapping and distinct roles of CDPK family members in the pre-erythrocytic stages of the rodent malaria parasite. Plasmodium berghei. PLoS Pathog 16:e1008131. doi: 10.1371/journal. ppat. 1008131

Govindasamy, K., Jebiwott, S., Jaijyan, D. K., Davidow, A., Ojo, K. K., Van Voorhis, W. C., et al. (2016). Invasion of hepatocytes by Plasmodium sporozoites requires cGMP-dependent protein kinase and calcium dependent protein kinase 4. Mol. Microbiol. 102, 349-363. doi: 10.1111/mmi. 13466

Govindasamy, K., Khan, R., Snyder, M., Lou, H. J., Du, P., Kudyba, H. M., et al. (2019). Plasmodium falciparum cyclic GMP-Dependent protein kinase interacts with a subunit of the parasite proteasome. Infect. Immun. 87:e00523-18. doi: 10.1128/IAI.00523-18

Gurnett, A. M., Liberator, P. A., Dulski, P. M., Salowe, S. P., Donald, R. G., Anderson, J. W., et al. (2002). Purification and molecular characterization of cGMP-dependent protein kinase from Apicomplexan parasites. a novel chemotherapeutic target. J. Biol. Chem. 277, 15913-15922. doi: 10.1074/jbc. $\mathrm{m} 108393200$

Jia, Y., Marq, J. B., Bisio, H., Jacot, D., Mueller, C., Yu, L., et al. (2017). Crosstalk between PKA and PKG controls pH-dependent host cell egress of Toxoplasma gondii. EMBO J. 36, 3250-3267. doi: 10.15252/embj.201796794

Jimenez-Diaz, M. B., Mulet, T., Viera, S., Gomez, V., Garuti, H., Ibanez, J., et al. (2009). Improved murine model of malaria using Plasmodium falciparum competent strains and non-myelodepleted NOD-scid IL2Rgammanull mice engrafted with human erythrocytes. Antimicrob. Agents Chemother. 53, 45334536. doi: 10.1128/aac.00519-09

Kim, J. J., Flueck, C., Franz, E., Sanabria-Figueroa, E., Thompson, E., Lorenz, R., et al. (2015). Crystal structures of the carboxyl cGMP binding domain of the Plasmodium falciparum cGMP-dependent protein kinase reveal a novel capping triad crucial for merozoite egress. PLoS Pathog 11:e1004639. doi: 10. 1371/journal.ppat.1004639

Mahmood, S. U., Cheng, H., Tummalapalli, S. R., Chakrasali, R., Bheemanaboina, R. R. Y., Kreiss, T., et al. (2020). Discovery of isoxazolyl-based inhibitors of Plasmodium falciparum cGMP-dependent protein kinase. RSC Med. Chem. 11, 98-101. doi: 10.1039/c9md00511k

Matralis, A. N., Malik, A., Penzo, M., Moreno, I., Almela, M. J., Camino, I., et al. (2019). Development of chemical entities endowed with potent fast-killing properties against Plasmodium falciparum malaria parasites. J. Med. Chem. 62, 9217-9235. doi: 10.1021/acs.jmedchem.9b01099

McRobert, L., Taylor, C. J., Deng, W., Fivelman, Q. L., Cummings, R. M., Polley, S. D., et al. (2008). Gametogenesis in malaria parasites is mediated by the cGMP-dependent protein kinase. PLoS Biol. 6:e139. doi: 10.1371/journal.pbio. 0060139

Moon, R. W., Taylor, C. J., Bex, C., Schepers, R., Goulding, D., Janse, C. J., et al. (2009). A cyclic GMP signalling module that regulates gliding motility in a malaria parasite. PLoS Pathog 5:e1000599. doi: 10.1371/journal.ppat.1000599

Panchal, D., and Bhanot, P. (2010). Activity of a trisubstituted pyrrole in inhibiting sporozoite invasion and blocking malaria infection. Antimicrob. Agents Chemother. 54, 4269-4274. doi: 10.1128/aac.00420-10

Patel, A., Perrin, A. J., Flynn, H. R., Bisson, C., Withers-Martinez, C., Treeck, M., et al. (2019). Cyclic AMP signalling controls key components of malaria parasite host cell invasion machinery. PLoS Biol. 17:e3000264. doi: 10.1371/journal.pbio. 3000264

Penzo, M., de Las Heras-Duena, L., Mata-Cantero, L., Diaz-Hernandez, B., Vazquez-Muniz, M. J., Ghidelli-Disse, S., et al. (2019). High-throughput screening of the Plasmodium falciparum cGMP-dependent protein kinase identified a thiazole scaffold which kills erythrocytic and sexual stage parasites. Sci, Rep. 9:7005.

Scribner, A., Meitz, S., Fisher, M., Wyvratt, M., Leavitt, P., Liberator, P., et al. (2008). Synthesis and biological activity of anticoccidial agents: 5,6-diarylimidazo[2,1b][1,3]thiazoles. Bioorganic Med. Chem. Lett. 18, 5263-5267. doi: 10.1016/j. bmcl.2008.08.063

Taylor, H. M., McRobert, L., Grainger, M., Sicard, A., Dluzewski, A. R., Hopp, C. S., et al. (2010). The malaria parasite cyclic GMP-dependent protein kinase plays a central role in blood-stage schizogony. Eukaryot. Cell 9, 37-45. doi: 10.1128/ec.00186-09

Tsagris, D. J., Birchall, K., Bouloc, N., Large, J. M., Merritt, A., Smiljanic-Hurley, E., et al. (2018). Trisubstituted thiazoles as potent and selective inhibitors of Plasmodium falciparum protein kinase G (PfPKG). Bioorganic Med. Chem. Lett. 28, 3168-3173. doi: 10.1016/j.bmcl.2018.08.028

Vanaerschot, M., Murithi, J. M., Pasaje, C. F. A., Ghidelli-Disse, S., Dwomoh, L., Bird, M., et al. (2020). Inhibition of Resistance-Refractory P. Falciparum kinase PKG delivers prophylactic. blood stage, and transmission-blocking antiplasmodial activity. Cell Chem. Biol. 27:e8.

Wall, M. E., Francis, S. H., Corbin, J. D., Grimes, K., Richie-Jannetta, R., Kotera, J., et al. (2003). Mechanisms associated with cGMP binding and activation of cGMP-dependent protein kinase. Proc. Natl. Acad. Sci. U S A. 100, 2380-2385. doi: $10.1073 /$ pnas. 0534892100

Wells, T. N., Hooft, van Huijsduijnen, R., and Van Voorhis, W. C. (2015). Malaria medicines: a glass half full? Nat. Rev. Drug. Discov. 14, 424-442. doi: 10.1038/ $\operatorname{nrd} 4573$

Wilde, M. L., Triglia, T., Marapana, D., Thompson, J. K., Kouzmitchev, A. A., Bullen, H. E., et al. (2019). Protein kinase a is essential for invasion of Plasmodium falciparum into human erythrocytes. mBio 10:e01972-19. doi: 10. 1128/mBio.01972-19

Zhang, R., and Monsma, F. (2010). Fluorescence-based thermal shift assays. Curr. Opin. Drug Discov. Dev. 13, 389-402.

Conflict of Interest: The authors declare that the research was conducted in the absence of any commercial or financial relationships that could be construed as a potential conflict of interest.

Copyright (C) 2021 Rotella, Siekierka and Bhanot. This is an open-access article distributed under the terms of the Creative Commons Attribution License (CC BY). The use, distribution or reproduction in other forums is permitted, provided the original author(s) and the copyright owner(s) are credited and that the original publication in this journal is cited, in accordance with accepted academic practice. No use, distribution or reproduction is permitted which does not comply with these terms. 